\title{
Slant perception and binocular brightness differences: Some aftereffects of viewing apparent and objective surface slants
}

\author{
JAMES T. WALKER \\ University of Missouri-St. Louis, St. Louis, Missouri 63121
}

\begin{abstract}
Placing a neutral-density filter in front of one eye produces two kinds of distortion in the perceived slant of a binocularly viewed rotating disk: (1) the top or the bottom of a disk rotating in a frontoparallel plane appears displaced toward or away from the observer, depending on the direction of rotation and whether the left or right eye is filtered; and (2) the left or right side of such disk-rotating or stationary-appears closer, depending on whether the left or right eye is filtered. The Pulfrich phenomenon accounts for the first variety of apparent slant, and the Venetian blind effect accounts for the second. Viewing the apparent slant of the rotating disk produces an aftereffect of slant in the third dimension which is greater than the aftereffect of viewing an objective slant of the same direction and magnitude.
\end{abstract}

Visual surfaces comprise a large part of the visual world. Most of the features of visual space perception-lines, angles, and edges, for example-are properties of surfaces. Indeed, Gibson (1950a) has argued that visual space perception is reducible to the perception of visual surfaces.

The slant of a surface, its orientation relative to the observer, is an important variable in a number of perceptual processes, such as depth perception, size constancy, and shape constancy (Gibson, 1950a, b). Some of the monocular cues for slant perception are visual texture gradients, linear perspective, and especially the shape of a surface as determined by its edges (Gibson, 1950b; Farné, 1970). Binocular disparities associated with edges and with visual texture elements also provide powerful cues for slant perception (Ogle, 1964).

Prolonged viewing of a slanted surface produces a negative aftereffect of slant in the third dimension (Bergman \& Gibson, 1959; Köhler \& Emery, 1947; Wenderoth, 1970). For example, after inspecting a slanted surface having its lower edge nearer the observer, an objectively frontoparallel surface appears slanted in the opposite direction. In this respect, visual slant perception is similar to several other perceptual processes where negative aftereffects also occur, such as visual and kinesthetic figural aftereffects (Köhler \& Dinnerstein, 1947; Köhler \& Wallach, 1944).

Viewing an apparently slanting surface in a stereoscope also produces a negative aftereffect of slant

This project was supported in part by a University of Missouri Summer Research Fellowship. A version of this paper was presented to the Eastern Psychological Association, Boston, Massachusetts, April 1972.
(Köhler \& Emery, 1947). In a random-dot stereogram, viewing a frontoparallel surface apparently displaced toward or away from the observer relative to the plane of fixation produces an opposite displacement in a subsequently presented surface lying in the plane of fixation (Blakemore \& Julesz, 1971). Thus, apparent displacements in depth, as well as objective displacements, produce negative aftereffects in the third dimension.

Apparent surface slants can also be produced under binocular viewing conditions by dimming the image in one eye relative to the other (Békésy, 1970; Cibis \& Haber, 1951; Walker, 1968). Consider a disk rotating at $12 \mathrm{rpm}$ in a frontoparallel plane (see Figure 1A). A stationary ring surrounds the disk. When the observer views the rotating disk binocularly with a neutral-density filter covering one eye, the disk appears strongly slanted in the third dimension (see Figure 1B). Filtering the left eye and rotating the disk clockwise makes the bottom of the disk appear nearer and the top farther away; switching the filter to the other eye reverses the direction of apparent slant, and reversing the direction of rotation while keeping the same eye filtered also reverses the apparent slant. With the left eye filtered, the left side of the disk appears closer to the observer-whether the disk is stationary or rotating in either directionand with the right eye filtered, the right side appears closer (see Figure 1C). Thus, the apparent slant has a vertical component which depends on direction of rotation and a horizontal component which does not.

The Pulfrich phenomenon (Lit, 1949; Pulfrich, 1922) accounts for the vertical component of the apparent slant of the rotating disk (Walker, 1968). Suppose the observer views an object moving horizontally from left to right in a frontoparallel plane. A 


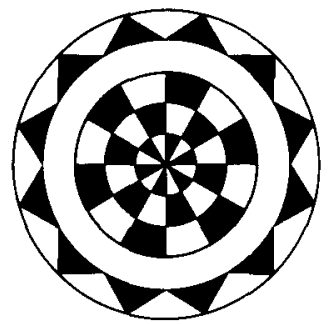

A. DISK AND RING

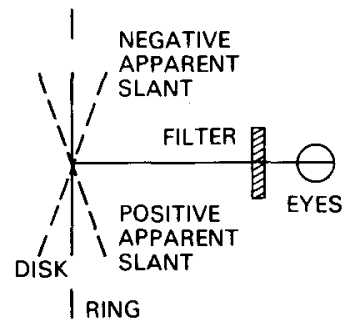

B. SIDE VIEW

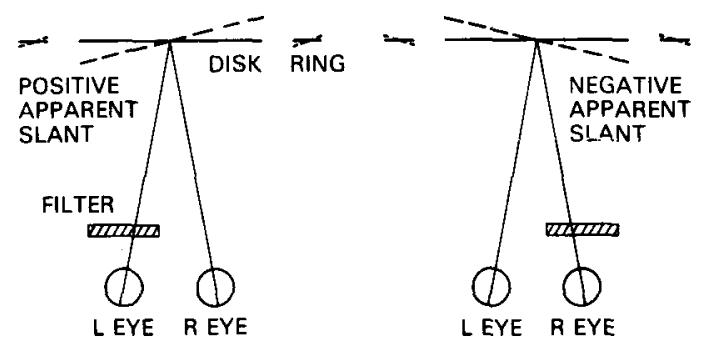

C. PLAN VIEWS

Figure 1. (A) Observer's view. Disk and ring were seen against a black background not shown here. (B) Side view, not to scale. Dashed lines show positive and negative apparent vertical slants of rotating disk. (C) Plan views, not to scale, showing positive and negative apparent horizontal slants of rotating or stationary disk and surrounding ring.

stationary fixation point is located directly below the path of the moving object. The observer fixates the stationary point and observes the moving object with the left eye filtered. The dimmer image in the filtered eye has a longer latency than the brighter image in the unfiltered eye, and thus the apparent position of the moving object as seen by the left eye lags behind its apparent position as seen by the right eye. The moving object is seen with divergent disparity, and thus appears farther away from the observer than the stationary fixation point, in accordance with the principles of binocular vision. An object moving from right to left viewed with the left eye filtered is seen with convergent disparity and appears nearer to the observer than a stationary fixation point. In the case of a disk rotating clockwise in a frontoparallel plane, the top of the disk moves from left to right and the bottom from right to left. With the left eye filtered, the bottom of such a rotating disk appears closer to the observer.

The horizontal component of the apparent slant can be explained by noting that the brighter image in the unfiltered eye is effectively larger than the dimmer image in the filtered eye, due to the greater spread of the brighter image (Békésy, 1970; Cibis \& Haber, 1951). The horizontal disparity between the dimmer and brighter image of an objectively frontoparallel disk reproduces the binocular disparity associated with an objectively horizontally slanted surface. In the case of a surface objectively slanted toward the observer's left or right, the nearer edge of the surface is located closer to the eye having the smaller horizontal projection in the retinal image. In the case of the apparent slant, the left edge of an objectively frontoparallel surface appears closer to the observer when the left eye is filtered, and the right edge appears closer when the right eye is filtered. The left and right sides of the ring also appear slanted, as in Figure 1C, but the ring does not appear slanted as a unit. In another context, comparable horizontal slants have been called the Venetian blind effect (Cibis \& Haber, 1951).

Inspecting the apparent slant of the rotating disk produces a negative aftereffect. The present study was designed to measure that aftereffect, to compare the aftereffects of inspecting apparent and objective slants of equal magnitude, and to assess the effects of fixating vs. visually tracking the rotating disk during the inspection period. With the earliest demonstration of visual aftereffects in the third dimension, Köhler and Emery (1947) raised the question of whether those aftereffects were a new phenomenon, or whether they resulted from figural aftereffects in the first two dimensions of visual space. The present study also addresses that issue.

\section{EXPERIMENT 1}

This experiment was designed (1) to measure the aftereffects of inspecting the apparent slant of the rotating disk, and (2) to measure the aftereffects of inspecting objective slants of equal magnitude. In order to equate the magnitudes of those two varieties of inspection stimuli for each observer, the magnitude of apparent slant was first measured. Repeated measures of the aftereffects of inspecting the same degree of objective and apparent vertical slants were then made for each observer.

\section{Method}

Observers. Twenty-four men, introductory psychology students at the University of Missouri-St. Louis, participated as a course requirement. Ten potential observers were excluded for reasons explained below. Observers ranged in age from 18 to 43 years, with a mean and standard deviation of 21.0 and 5.29 years. Those persons excluded from the experiment ranged in age from 18 to 26 years, with a mean and standard deviation of 20.5 and 2.55 years; they did not differ significantly in age from the observers used in the experiment, $\mathrm{t}(32)=.28$.

Apparatus. The visual display consisted of a disk and surrounding ring presented at eye level in a frontoparallel plane (see Figure 1A), The display was presented at a distance of $6 \mathrm{ft}(183 \mathrm{~cm})$ from the observer. The disk was $17.8 \mathrm{~cm}$ in diameter, and subtended a visual angle of $5.57^{\circ}$. The ring was separated from the disk by an open annulus. The inner and outer diameters of the ring were, respectively, 23.9 and $29.8 \mathrm{~cm}$; those diameters, respectively, subtended visual angles of $7.50^{\circ}$ and $9.33^{\circ}$.

The disk could be rotated at $12 \mathrm{rpm}$ by a tachometer-feedbackcontrolled dc servomotor. The surrounding ring always remained stationary. The disk was suspended in a system of yokes and bearings so that its slant could be adjusted, whether the disk was rotating or stationary. Regardless of its slant, the center of the disk remained in coincidence with the center of the ring. The observer 
adjusted the horizontal slant by turning a horizontal wheel which turned the disk about its vertical diameter. A vertical wheel adjusted the vertical slant by turning the disk about its horizontal diameter. Horizontal and vertical adjustments could be made simultaneously. In all cases, a null procedure was used to measure the apparent slant of the disk-that is, the observer adjusted the objective slant to compensate for the apparent slant so that the disk appeared to lie in the same plane as the stationary ring.

The disk and ring were housed in a black enclosure and were viewed through a circular aperture. A background of black velvet completely hid the structural members of the apparatus. The display was lighted by a 100 -W slide projector powered by a constantvoltage transformer. The projector was located below the observer's line of sight. A circular aperture in the slide plane of the projector provided a sharply defined area of illumination which lighted the disk and ring. Stray light from the projector lens was greatly reduced by placing a circular aperture $7.6 \mathrm{~cm}$ in front of the lens.

The disk and ring were made of white paper having a highly reflective matte surface and black flocked paper. The luminances of the white and black areas, measured with a Macbeth illuminometer, were 18.4 and $.11 \mathrm{~mL}$. The visual display thus had a very high contrast ratio' of .99 . The observers always viewed the ring and disk through two Wratten neutral-density filters placed in a face mask. One eye was always filtered with a density of 1.00 and the other with a density of 2.00 ; the luminances of the white areas of the display seen by the less heavily and more heavily filtered eyes were, respectively, 1.84 and $.18 \mathrm{~mL}$.

Sighting dominance and acuity measures. Sighting dominance was assessed by having each observer align two vertical crosshairs in a simple sighting device with a target several feet away. Three trials were given. A score of 0 or 1 was given for each trial depending on whether the left or the right eye was used. Eye dominance scores ranged from 0 to 3 with a mean and standard deviation of 2.08 and 1.25 . The mean dominance score differed significantly from 1.5 , the value expected if neither eye were dominant, $\mathrm{t}(23)=2.29 ; \mathrm{p}<.05$. Thus, for most observers the right eye was dominant.

Acuity was measured with a Bausch \& Lomb Ortho-Rater. Far binocular acuity (1/visual angle in minutes) ranged from .8 to at least 1.2 , with a mean and standard deviation of 1.06 and .11 . Stereoacuity ranged from at least as good as 9.7 to 32 seconds of arc, with a mean and standard deviation of 21.35 and 8.11. One potential observer who was blind in one eye was excluded; four persons having stereoacuity scores of 2 or less, indicating stereoacuities of 83 seconds of arc or more, were also excluded.

Practice trials and control measures. For half the observers, the left eye was more heavily filtered, and for the other half, the right was more heavily filtered. Directions of rotation, clockwise and counterclockwise, were balanced across filter conditions. Each observer was tested under one of the four combinations of filter conditions and directions of rotation.

For demonstration purposes, the disk was first shown stationary. The disk was slanted $20^{\circ}$ vertically and horizontally, with the bottom edge and the right edge closer to the observer. Those vertical and horizontal slants are defined, respectively, as positive and negative (see Figure 1). The experimenter demonstrated the adjustment of the disk and then instructed the observer to adjust the disk ". . so that it lines up even with the ring as nearly as possible." One practice trial was given at this point. Each observer turned the vertical adjustment wheel with the preferred hand and the horizontal wheel with the other hand. The observer was instructed to look directly at the top and bottom and left and right edges of the disk while making those adjustments. The observer then made a practice adjustment of the rotating disk. In the next two practice trials, the disk was stationary and the observer was instructed to adjust the disk while fixating its center. The starting positions for those trials were (1) $\mathrm{H}-10^{\circ}, \mathrm{V} 10^{\circ}$, and (2) $\mathrm{H} 10^{\circ}, \mathrm{V}-10^{\circ}$. After those trials, the room lights were turned off and from that point on the room remained dark. The observer was then given six timed trials in which he had $15 \mathrm{sec}$ to adjust the stationary disk while fixating its center. The length of each trial was controlled by an electrically operated shutter. A neon warning light came on $10 \mathrm{sec}$ before the shutter opened for each trial. As soon as the shutter opened, the experimenter set the slant of the disk to one of the starting positions used in the two preceding untimed practice trials. The order of starting positions in the block of six timed trials was (2)(1)(1) (2) (2) (1), as described above. There was an interval of $30 \mathrm{sec}$ between trials. The horizontal and vertical slant components of the disk were read to the nearest $1 / 2^{\circ}$ on graduated circles. The first two of the six preceding trials were discarded, and the last four were used as control measures.

Since the aftereffects to be measured later in the experiment were expected to dissipate with time, it was considered necessary for observers to complete their adjustments within the allotted $15 \mathrm{sec}$. Two persons were unable to do so and were replaced by other observers.

Measures of apparent slant. A block of four untimed measures was made with the observer fixating the center of the rotating disk. On the first trial, the starting position was in the frontoparallel plane. For the starting positions on the next two trials, the experimenter set the magnitudes of the horizontal and vertical slants about $10^{\circ}$ greater than the observer's adjustments on the preceding trial. For the last trial, the magnitude was set about $10^{\circ}$ less than the observer's preceding adjustment. Thus, the starting positions on those four trials were presented in A D D A order. The experimenter calculated the mean vertical slant of the rotating disk in order to equate objective and apparent slants in the aftereffect phase of the experiment.

One observer was replaced because of the low magnitude, $.75^{\circ}$, of his apparent vertical slant. For the 24 observers who were retained, magnitudes of apparent vertical slant ranged from $7.12^{\circ}$ to $22.75^{\circ}$.

Aftereffect measures. Aftereffects were measured for each observer after inspecting objective and apparent slants. Under the apparent condition, the disk was rotating at $12 \mathrm{rpm}$ in the frontoparallel plane. A neon warning light came on $10 \mathrm{sec}$ before the shutter opened for each inspection period. Neutral-density filters were always in place, and thus the disk appeared slanted. The observer fixated the center of the rotating disk for an inspection period of $45 \mathrm{sec}$. The disk then stopped, and a $15-\mathrm{sec}$ test period began. The experimenter moved the stationary disk to one of the starting positions described above in connection with control measures, and the observer then adjusted the disk. At the end of the test period, the shutter closed. There was an intertrial interval of $60 \mathrm{sec}$ before the beginning of the next inspection period. A block of four inspection periods, each followed by a test period, was presented under the same inspection condition (apparent or objective slant). Orders of inspection conditions were balanced across directions of rotation and across filter positions (left or right eye more heavily filtered). There was a 2 -min interval between blocks of apparent and objective inspection conditions.

Under the objective condition, the disk was stationary. For each observer, the vertical slant of the stationary disk was set equal to that observer's previously measured apparent vertical slant of the rotating disk. Horizontal slants were set equal to zero under both conditions. Thus, under the objective condition, only the vertical slant was an objective slant; an apparent horizontal slant was present under both objective and apparent conditions in the stationary as well as the rotating disk because the neutraldensity filters were always in place.

During the aftereffect phase of the experiment, two observers were replaced because of their failure to follow instructions.

\section{Results}

Control measures. As expected, filtering one eye more heavily had no significant effect on the apparent vertical slant of the stationary disk. With the heavier filter over the left and right eye, respectively, the mean apparent vertical slants were $-.33^{\circ}$ and $.17^{\circ}$; 
those means did not differ significantly, $t(22)=.63$, nor did the combined mean for both filter positions, $-.08^{\circ}$, differ significantly from zero, $t(23)=.21$.

The apparent horizontal slant of the stationary disk was affected by filter position. With the left and right eye, respectively, more heavily filtered, the mean horizontal slants, $.04^{\circ}$ and $-4.04^{\circ}$, were significantly different, $\mathrm{t}(22)=3.63, \mathrm{p}<.01$. With the right eye more heavily filtered, the right side of the disk appeared closer to the observer, as shown by the negative apparent horizontal slant of $-4.04^{\circ}$, which differed significantly from zero, $t(11)=4.24$, $p<.01$. With the left eye more heavily filtered, the left side of the disk should have appeared closer to the observer, and while the positive slant of $.04^{\circ}$ was in the expected direction, that slant did not differ significantly from zero, $t(11)=.07$. The combined mean horizontal slant for both filter positions was found by assigning a positive sign to slants that were in the expected direction; that mean, $2.04^{\circ}$, differed significantly from zero, $\mathrm{t}(23)=2.96, \mathrm{p}<.01$.

The possibility exists that differences in eye dominance or stereoacuity might have contributed to the observed differences between the two groups of observers having the left and the right eye more heavily filtered. But the mean eye-dominance scores for those groups were not significantly different, 2.17 and $2.00, \mathrm{t}(22)=.33$; nor were the stereoacuity measures, 20.87 and $21.83 \mathrm{sec}$ of arc, $t(22)=$ .14.

Apparent slant of the rotating disk. The horizontal and vertical slant components are shown in Figure $2 \mathrm{~A}$. Measures of horizontal slant were subjected to a two-way analysis of variance where the factors were direction of rotation (clockwise or counterclockwise) and filter position (left or right eye more heavily filtered). For the horizontal slant, there was no significant effect of rotation direction and no significant interaction, both Fs $<1$, but the effect of filter position was highly significant, $F(1,20)=37.74$, $p<.001$. With the left eye more heavily filtered, the mean horizontal slant was $3.51^{\circ}, t(11)=4.73$, $\mathrm{p}<.01$; and with the right, the mean was $-6.02^{\circ}$, $\mathrm{t}(11)=4.52, \mathrm{p}<.01$. Thus, the apparent horizontal slant of the rotating disk was determined by filter position and was independent of direction of rotation. The combined mean apparent horizontal slant for both filter positions was found by assigning a positive sign to each adjustment that was made in the expected direction; that mean, $4.77^{\circ}$, differed significantly from zero, $t(23)=6.03, p<.001$, and also differed significantly from the mean horizontal control measure, $2.04^{\circ}$, for the stationary disk, $\mathrm{t}(23)=2.90, \mathrm{p}<.01$.

The apparent vertical slant of the rotating disk was found by subtracting the vertical control adjustments of the stationary disk from the vertical adjustments of the rotating disk. The resulting measures of
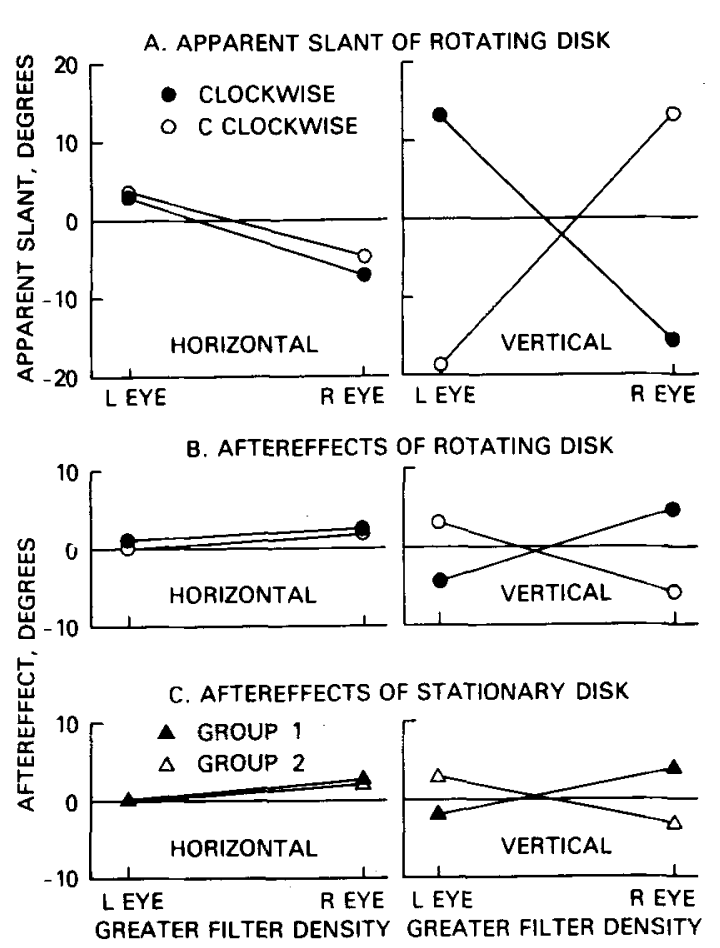

Figure 2. (A) Apparent horizontal and vertical slants of rotating disk. (B) Aftereffects of inspecting apparent slants of rotating disk. (C) Aftereffects of inspecting apparent horizontal and objective vertical slants of stationary disk. Groups 1 and 2 were, respectively, the observers who viewed the disk rotating clockwise and counterclockwise under the rotation conditions. Each observer viewed an objective vertical slant of the stationary disk equal in magnitude and direction (positive or negative) to that observer's apparent slant of the rotating disk.

apparent vertical slant were subjected to a two-way analysis of variance. As expected, there were no main effects of rotation direction or filter position, each $F(1,20)<1$, but there was a highly significant interaction, also as expected, $F(1,20)=309.61, p<.001$. The nature of that interaction can be seen in Figure 2. For each of the four combinations of filter position and direction of rotation, the mean apparent vertical slant was in the expected direction and was significantly different from zero, each $t(5)>6, p<.01$. The mean magnitude of apparent vertical slant across all conditions was $15.44^{\circ}$, highly significantly different from zero, $t(23)=15.93, p<.001$.

Aftereffects. Figure 2B shows the aftereffects of inspecting the rotating disk. The aftereffects of inspecting the stationary disk are shown in Figure $2 \mathrm{C}$, where Groups 1 and 2 are, respectively, the observers who viewed the disk rotating clockwise and counterclockwise under the rotation condition shown in Figure 2B.

Horizontal aftereffect measures were found for each observer by subtracing his mean control adjustment from his mean test adjustment. The measures were subjected to a three-way analysis of variance where the factors were direction of rotation (clock- 
wise or counterclockwise), filter position (left or right eye more heavily filtered), and rotation condition (rotating or stationary). There were repeated measures on the last factor. Only one effect approached significance, that of filter position, $F(1,20)=3.81, p<.10$. For the rotating disk, with the left eye more heavily filtered, the combined mean horizontal aftereffect for both directions of rotation was $.60^{\circ}$, not significantly different from zero, $t(11)=1.16$; with the right eye more heavily filtered, the combined mean was $1.85^{\circ}, \mathrm{t}(11)=2.203$, $\mathrm{p}<.05$. For the stationary disk, with the left eye more heavily filtered, the combined mean horizontal aftereffect was not significantly different from zero, $.06^{\circ}, t(11)=.1 \mathrm{i}$; with the right eye more heavily filtered, the combined mean was $2.22^{\circ}, t(11)=3.78$, $p<.01$. Thus, the apparent horizontal slant of the disk, whether rotating or stationary, produced an aftereffect only when the right eye was more heavily filtered.

Vertical aftereffect measures were also found for each observer by subtracting control from test adjustments, and a three-way analysis of variance was carried out as described above. There was no significant main effect of rotation direction (clockwise or counterclockwise), filter position (left or right eye more heavily filtered), or rotation condition (rotating or stationary). There was a highly significant Rotation Direction by Filter Position interaction, $F(1,24)=$ $64.37, \mathrm{p}<.001$. For each of the four combinations of rotation direction and filter position, the vertical aftereffects of inspecting the rotating disk (Figure $2 \mathrm{~B}$ ) and the stationary disk (Figure $2 \mathrm{C}$ ) were all in the expected direction and all significantly different from zero by one-tailed tests, each $t(5)>2.06$, $\mathrm{p}<.05$. Positive apparent slants of the rotating disk produced aftereffects of negative slant, and negative apparent slants produced aftereffects of positive slant. In the stationary disk, positive and negative objective slants produced analogous aftereffects. Thus, positive and negative slants both produced "negative" aftereffects in the sense that after the inspection period, the disk appeared to slant in the opposite direction from the inspection slant.

There was also a significant Rotation Direction by Filter Position by Rotation Condition interaction in the vertical aftereffect measures, $F(1,20)=10.55$, $p<.01$. Given the two-way interaction described above, the triple interaction implies that the Filter Position by Rotation Direction interaction differed across levels of rotation condition (rotating or stationary). The nature of the difference between the two-way interaction for the rotating and stationary disk can be seen in Figures 2B and 2C. Under all four combinations of filter position and rotation direction, the rotating disk produced greater aftereffects, in terms of absolute magnitude, than the stationary disk. Assigning a positive sign to after- effects that were in the expected direction, the mean vertical aftereffects under the rotating and stationary conditions were $4.57^{\circ}$ and $3.11^{\circ}$; those aftereffects differed significantly from each other, $t(23)=3.25$, $p<.01$, and also from zero, each $t(23)>6$, $\mathrm{p}<.001$.

\section{EXPERIMENT 2}

In the previous experiment, the observers fixated the center of the disk under all of the experimental conditions. Preliminary observations suggested that the apparent slant of the rotating disk was greater under conditions of visual tracking, that is, where the observer's eyes followed a point on the edge of the disk. The present experiment was designed to measure the apparent slant of the rotating disk, and also the aftereffects of inspecting the apparent slant, under conditions of visual tracking.

\section{Method}

Observers. Twelve men, introductory psychology students, participated as a course requirement. Observers ranged in age from 19 to 30 years with a mean and standard deviation of 23.08 and 4.03 years. Sighting dominance and visual acuity were measured as in Experiment 1. Eye dominance scores ranged from 0 to 3 with a mean and standard deviation of 1.83 and 1.34 . The mean dominance score did not differ significantly from 1.50 , the value expected if neither eye were dominant, $t(11)=.86$.

Far binocular acuity (1/visual angle in minutes) ranged from .8 to at least 1.2 , with a mean and standard deviation of 1.06 and .11, exactly as in Experiment 1 . Stereoacuity ranged from at least as good as 9.7 to 43 seconds of arc, with a mean and standard deviation of 18.68 and 11.05. Three potential observers having stereoacuity scores of 2 or less, indicating stereoacuities of 83 seconds of arc or more, were excluded. Those excluded from the experiment ranged in age from 19 to 28 years, with a mean and standard deviation of 23.67 and 4.51 years.

Apparatus. The apparatus was the same as that used in Experiment 1 .

Procedure. The observer always viewed the visual display through two Wratten neutral-density filters. The left eye was always filtered with a density of 2.00 and the right with a density of 1.00. The procedures were the same as those used in Experiment 1 , with two exceptions. In the present experiment, the observers were instructed: (1) to visually track a point on the edge as they adjusted or inspected the rotating disk; and (2) to look directly at the top and bottom and left and right edges as they adjusted the stationary disk. The procedure for viewing and adjusting the stationary disk during control and test trials in the present experiment was designed to be compatible with the procedure used for viewing and adjusting the rotating disk.

The apparent slant was measured with the disk rotating clockwise for half the observers and counterclockwise for the other half. Repeated measures of the aftereffects of inspecting clockwise and counterclockwise rotation were made for each observer. A block of four inspection periods, each followed by a test period, was given for each direction of rotation. Orders of rotation directions were balanced across observers. For each observer, the direction of rotation for the first block of aftereffect measures was the opposite of the direction previously used to measure the apparent slant of the rotating disk.

\section{Results}

In the present experiment, the left eye was always more heavily filtered for all 12 observers. Wherever 
comparisons were made with the results of Experiment 1 , those comparisons utilized only the 12 observers in that experiment for whom the left eye was more heavily filtered. The observers in Experiment 1 did not differ significantly from the observers in Experiment 2 in stereoacuity, 20.87 and 18.68 seconds of arc, respectively, $\mathrm{t}(22)=.52$, or in eye dominance, 2.17 and $1.83, \mathrm{t}(22)=.64$. Thus, it is unlikely that differences in stereoacuity or eye dominance could have contributed significantly to any of the observed differences between the two experiments which are described below.

Control measures. The mean apparent vertical slant of the stationary disk was not significantly different from zero, $-.34^{\circ}, t(11)=.63$. The mean apparent horizontal slant differed significantly from zero, $3.81^{\circ}, \mathrm{t}(11)=3.13, \mathrm{p}<.01$, and also from the results of Experiment $1, \mathrm{t}(22)=3.13, \mathrm{p}<.01$. In the present experiment, observers looked at the edges of the disk as they made their adjustments, but in Experiment 1, they fixated the center of the disk. The relatively small apparent horizontal slant of the stationary disk may have been more difficult to see in peripheral vision under the conditions of Experiment 1.

Apparent slant of rotating disk. For clockwise and counterclockwise rotation, the mean apparent horizontal slants did not differ significantly from each other, $9.81^{\circ}$ and $7.87^{\circ}, t(10)=1.25, p>.20$; but each mean was significantly different from zero, $\mathrm{t}(5)=18.18, \mathrm{p}<.001$, and $\mathrm{t}(5)=5.41, \mathrm{p}<.01$. The combined mean for both directions of rotation, $8.84^{\circ}$, was significantly greater than the corresponding mean in Experiment $1,3.51^{\circ}, \mathrm{t}(22)=4.90$, $\mathrm{p}<.001$. Comparisons between Experiments 1 and 2 are shown in Figure 3.

The mean apparent vertical slants for clockwise and counterclockwise rotation were significantly different from each other, $-21.46^{\circ}$ and $20.08^{\circ}$, $t(10)=12.64, p<.001$; and each was significantly different from zero, each $t(5)>8, p<.001$. The combined mean apparent vertical slant was found for both directions of rotation by assigning a positive sign to all values which were in the expected direction; that mean, $20.77^{\circ}$, was significantly greater than the corresponding mean in Experiment $1,15.99^{\circ}, \mathrm{t}(22)=2.19, \mathrm{p}<.05$. Thus, the magnitudes of vertical as well as horizontal apparent slants were greater under the condition of visual tracking than under the condition of fixation.

Aftereffects. The mean horizontal aftereffects of inspecting clockwise and counterclockwise rotations did not differ significantly from each other, $-.42^{\circ}$ and $-1.04^{\circ}, \mathrm{t}(11)=1.43, \mathrm{p}<.20$. While those aftereffects were in the expected direction, the combined mean horizontal aftereffect did not differ significantly from zero, $-.73^{\circ}, t(11)=1.65, p<.10$, one-tailed. The mean vertical aftereffects for clock-

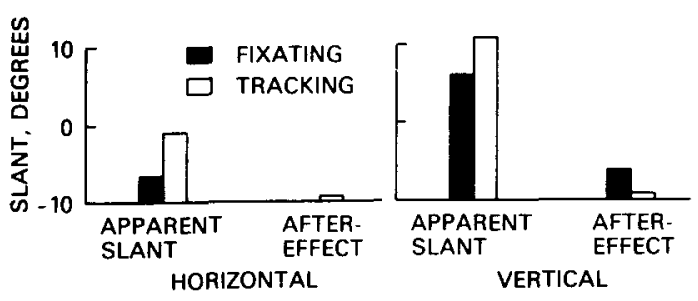

Figure 3. Effects of fixating (Experiment 1) and visual tracking (Bxperiment 2) on the apparent siant of the rotating disk, and on the aftereffects produced by the apparent slant.

wise and counterclockwise rotations were in the expected directions, but those means did not differ significantly from each other, $-1.26^{\circ}$ and $.22^{\circ}$, $t(11)=1.78, p<.20$; nor did either mean differ significantly from zero, $t(11)=1.77, p<.20$, and $t(11)=.30$.

Although the tracking procedure in the present experiment produced greater horizontal and vertical apparent slants than the fixation procedure in Experiment 1, there were no significant aftereffects in the present experiment.

\section{DISCUSSION}

For the stationary disk, there was a positive or negative apparent horizontal slant, depending on whether the left or the right eye was more heavily filtered. The apparent horizontal slant of the rotating disk also depended on filter position, and while that slant was independent of rotation direction, the magnitude of the horizontal slant was greater for the rotating than for the stationary disk in both Experiments 1 and 2. Stereopsis is maintained better with eye movements than with steady fixation (Ogle, 1967), and it may be that moving a visual display serves the same function as moving the eyes over a stationary display insofar as maintaining stereopsis is concerned.

As expected, there was no apparent vertical slant of the stationary disk. The apparent vertical slant of the rotating disk depended on the interaction of filter position and direction of rotation. For the stationary and for the rotating disk, ali of the apparent slants that were observed were greater in Experiment 2 than in Experiment 1 (see Figure 3). Two factors may account for the greater apparent slants in the second experiment: (1) visual tracking in Experiment 2 vs. steady fixation in Experiment 1; and (2) in Experiment 2, the edge of the disk and the surrounding ring were seen in central visionwhere acuity is better-vs. peripheral vision in Experiment 1 , because of the fixation procedure.

The apparent vertical slant of the rotating disk in Experiment 1 produced a significantly greater aftereffect than an objective slant equated for magnitude and direction. Since the apparent vertical slant was 
produced by the rotation of the disk, while the objectively slanting disk was always stationary, it may be-as suggested above-that stereopsis was maintained better in the rotating disk, possibly resulting in a greater aftereffect.

Although the visual tracking procedure in Experiment 2 produced greater apparent horizontal and vertical slants than the fixation procedure in Experiment 1 , there were no significant aftereffects in the second experiment while there were in the first (see Figure 3). Two possible explanations of the failure of the tracking procedure to produce aftereffects in Experiment 2 appear plausible: (1) As the observer visually tracked a point on the edge of the rotating disk, the image of the disk was spread across a circular area of the retina having a diameter about twice as large as the image of the steadily fixated disk. When the observer then adjusted the stationary disk during a test trial, a portion of the image of the ring fell on retinal areas previously adapted to the apparently slanting disk. ${ }^{2}$ If the ring, as well as the disk, were subject to an aftereffect, there might be no net apparent displacement between the two. An aftereffect might occur under such conditions, in the sense that both the ring and disk might be apparently displaced out of the frontoparallel plane, but it would not be possible to measure such an aftereffect by adjusting the disk relative to the ring. (2) Under the fixation condition, each point in the retinal image of the disk was continuously adapted to the apparent slant of the disk during the entire inspection period. But under the tracking condition, the image of the disk swept across a larger retinal area, within which each point was exposed to the disk during only a portion of the inspection period.

An earlier study may have some bearing on the second explanation offered above. Bergman and Gibson (1959) found no significant difference between the aftereffects of fixating and scanning a surface having a vertical slant of $15^{\circ}$; those aftereffects were, respectively, $2.55^{\circ}$ and $2.15^{\circ}$. Although there were many differences between that study and the present one, two differences appear crucial: the earlier inspection period was $4 \mathrm{~min}$, vs. $45 \mathrm{sec}$ in the present study; and in the earlier study, the slanted surface was viewed through a circular aperture which hid the edges of the surface. The aftereffects were measured by adjusting the surface so that it appeared to lie in the frontoparallel plane. For the reasons noted above. Bergman and Gibson's adjustment procedure may have yielded an aftereffect where the adjustment procedure in the present study might not.

In the earliest study of visual aftereffects in the third dimension, Köhler and Emery (1947) raised the question of whether those aftereffects were a new phenomenon, or whether they might be explained in terms of figural aftereffects in the first two dimensions of visual space. As a working hypothesis, Köhler and Emery devised the following tentative explanation. In binocular vision, each eye sees a slightly different image of a slanted surface-indeed, the horizontal spatial disparity between the two retinal images is the classical basis of stereopsis. ${ }^{3}$ In a single eye, the image of a slanted surface differs from the image of the same surface in the frontoparallel plane. If the observer inspects a slanted surface which is then adjusted to the frontoparallel plane, separate two-dimensional figural aftereffects might then occur in those regions of the visual system receiving monocular input from each eye. ${ }^{4}$ Those two-dimensional figural aftereffects would then displace each of the separate representations of an objectively frontoparallel surface in such a way as to make the surface appear slanted in the opposite direction from its previous slant.

Köhler and Emery (1947) went on to argue that the preceding explanation was untenable becauseamong other reasons-the displacements typically observed in two-dimensional figural aftereffects were not sufficiently large to account for the magnitude of their depth effect. A study using random-dot stereograms provides more convincing evidence against the role of two-dimensional figural aftereffects. Blakemore and Julesz (1971) demonstrated the displacement of frontoparallel surfaces toward or away from the observer after viewing farther or nearer surfaces. Since the depth planes in random-dot stereograms do not exist monocularly, threedimensional aftereffects in such stimuli cannot be explained in terms of two-dimensional aftereffects occurring separately in regions of the visual system receiving monocular input.

In the present study, the apparent vertical slant of the objectively frontoparallel rotating disk resulted from the longer latency of the moving image in the more heavily filtered eye. The latency difference between the moving contours in the dimmer and brighter image was translated into a spatial disparity between the locations of those contours in the two retinal images. Considering the two eyes separately, in each retinal image of the rotating disk the visual contours continuously swept across the image area. Since there was no opportunity for any particular area within the image to adapt to any particular orientation or location of the moving contours during the inspection period, there was no opportunity for two-dimensional figural aftereffects to develop in the two eyes in such a way as to produce a vertical slant aftereffect. Thus, the present study adds to the earlier evidence that aftereffects in the third dimension of visual space can occur independently of aftereffects in the first two dimensions.

The apparent horizontal slant of the objectively frontoparallel stationary and rotating disk occurred because of the horizontal disparity between the effectively larger image in the less heavily filtered eye and the effectively smaller image in the other eye. ${ }^{5}$ When the observer fixated the center of the 
disk, whether stationary or rotating, the edges of the disk always fell on the same area in each retinal image. Thus, there was an opportunity for twodimensional figural aftereffects to develop, which cannot be ruled out as a possible factor in the horizontal slant aftereffect observed in Experiment 1.

The apparently slanting surface produced by the rotating disk is quite compelling. The apparent slant can be readily produced by rotating a visually textured disk on a pursuit rotor (or on a phonograph turntable at 16 or $33 \mathrm{rpm}$ ) and binocularly viewing the disk with one lens of a pair of sunglasses in front of one eye. If an observer tracks a point on the rotating disk with a hand, and at the same time visually tracks the moving hand and disk, then the objectively horizontal turntable looks slanted and feels slanted as well. The conflict between distorted visual information and veridical tactual information is resolved in favor of vision, as in many other situations where visual capture occurs (e.g., Hay, Pick, \& Ikeda, 1965; Walker, 1971).

The apparently slanting surface of the rotating disk has much of the look-and feel-of an objectively slanted surface. The apparent vertical slant of the rotating disk produced a significantly greater aftereffect than an objective slant of the same magnitude. Thus, apparent and objective visual surfaces play comparable roles in some of the processes of visual space perception.

\section{REFERENCES}

BÉKf́sY, G. von. Apparent image rotation in stereoscopic vision: The unbalance of the pupils. Perception \& Psychophysics, 1970 , 8. 343-347.

Bergman, R., \& Grason, J. J. The negative after-effect of the perception of a surface slanted in the third dimension. American Journal of Psychology, 1959, 72, 363-374.

Blakemore, C., \& Julesz, B. Stereoscopic depth aftereffect produced without monocular cues. Science, 1971, 171, 286-289.

Cibis, P. A., \& Haber, H. Anisopia and perception of space. Journal of the Optical Society of America, 1951, 41, 676-683.

FARNÉ, M. The influence of margins on visual after-effects in the third dimension. Acta Psychologica, 1970, 32, 81-90.

Gibson, J. J. The perception of visual surfaces. American Journal of Psychology, 1950, 63, 367-384. (a)

GiBson, J. J. The perception of the visual world. Boston: Houghton Mifflin, 1950. (b)

Hay, J. C., Pick, H. L., JR., \& Ikeda, K. Visual capture produced by prism spectacies. Psychonomic Science, 1965, 2, 215-216.
Köhler, W., \& Dinnerstein, D. Figural aftereffects in kinesthesis. In Miscellanea psychologica: Albert Michotte. Louvain, Belgium: University of Louvain, 1947. Pp. 196-220.

KöLER, W., \& EMERY, D. A. Figural after-effects in the third dimension of visual space. American Journal of Psychology, $1947,60,159-201$

Köhler, W., \& Wallach, H. Figural after-effects: An investigation of visual processes. Proceedings of the American Philosophical Society, 1944, 88, 269-357.

LIT, A. The magnitude of the Pulfrich stereophenomenon as a function of binocular differences of intensity at various levels of illumination. American Journal of Psychology, 1949, 62, 159-181.

OGLE, K. N. Binocular vision. New York: Hafner, 1964.

OGLE, K. N. Some aspects of stereoscopic depth perception. Journal of the Optical Society of America, 1967, 57, 1073-1081.

Pulfrich, C. Die Stereoskopie im Dienste der isochromen und heterochromen Photometrie. Die Naturwissenschaften, 1922, 10, 553.564 .

Ross, J. Stereopsis by binocular delay. Nature, 1974, 248, 363-364.

Ross, J. The resources of binocular perception. Scientific American, 1976, 234(3), 80-86.

WALKER, J. T. The slanting disc effect: A kinetic visual stereophenomenon. Optometric Weekly, 1968, 59(46), 25-33.

WALEer, J. T. Visual capture in visual illusions. Perception \& Psychophysics, 1971, 10, 71-74.

Wenderoth, P. M. A visual spatial aftereffect of surface slant. American Journal of Psychology, 1970, 83, 576-590.

\section{NOTES}

1. The contrast tatio, $\mathrm{C}$, is defined by the following formula:

$$
C=\left(L_{\max }-L_{\min }\right) /\left(L_{\max }+L_{\min }\right)
$$

where $L_{\max }$ and $L_{\min }$ are, respectively, the maximum and minimum luminances in the visuai display.

2. This is not to suggest that slant aftereffects occur in the retina. Demonstrations of interocular transfer strongly implicate central mechanisms.

3. Ross $(1974,1976)$ has shown that a temporal disparity, a time difference, between some of the elements in a binocular display can give rise to stereopsis without first producing a spatial disparity. In the Pulfrich effect, a temporal disparity translates into a spatial disparity which then produces stereopsis in accordance with the classical principles of binocular vision. But in Ross's demonstrations, a temporal disparity per se gives rise to stereopsis.

4. The fact that interocular transfer occurs in two-dimensional figural aftereffects would seem to pose some difficulties for this explanation.

5. There is a vertical as well as horizontal disparity here. Although vertical disparity does not produce stereopsis directly, vertical disparity influences stereopsis through what Ogle (1964) has called the induced effect. In the present case, the induced effect opposes the effect of the horizontal disparity.

(Received for publication April 2, 1976; revision accepted August 30, 1976.) 